${ }^{1}$ Faculdade de Saúde Pública, USP. Av. Dr. Arnaldo 715, Cerqueira César. 01246904 São Paulo SP. alexdiasporto@usp.br

\title{
Partos cesáreos e a escolha da data de nascimento no Município de São Paulo
}

\author{
Caesarean sections and the choice of date of birth \\ in the Municipality of São Paulo, Brazil
}

\begin{abstract}
Brazil has one of the highest rates of cesarean births, and the number has been growing annually. The scope of this study was to test the possible consequences arising from this increase on the date of birth. The 1,933,137 live births that occurred in São Paulo, Brazil, from 2001 to 2010 were analyzed by type of delivery and exact date of birth. In 2001, 48.9\% of all births were by cesarean section, increasing to $56.8 \%$ in 2010. During th is period, the difference between the number of births occurring on Sundays (the day of the week with the lowest number) increased from 111.4 (95\%CI: 101.7 - 121.0) in 2001, to 143.1 (95\%CI: 135.0 - 151.3) in 2010. The two dates with the lowest number of births (Christmas day and the Day of the Dead), had a statistically significant $(p<0.05)$ difference from the other days of the year during most of the period (the exception being the Day of the Dead in 2009). The date with the highest number of births (International Women's Day) showed a statistically significant d ifference in 2005 and 2010. The increase in the number of cesarean births has had a significant impact on date of birth, which could have consequences for the administrative planning of health institutions.
\end{abstract}

Key words Live births, Cesarean sections, Health institutions, Date of birth
Resumo O Brasil tem uma das maiores taxas de partos cesáreos do mundo, e esse valor tem aumentado anualmente. O presente estudo teve como objetivo analisar possíveis mudanças na data de nascimento decorrentes desse aumento. Foram obtidos os dados dos 1.933.137 nascidos vivos ocorridos no Município de São Paulo entre 2001 e 2010, divididos segundo tipo de parto e data exata de nascimento. Em 2001, a taxa de cesarianas foi de 48,9\%, passando a 56,8\% em 2010. O dia da semana com menor número de nascimentos (domingo) aumentou a diferença em relação aos outros dias de 111,4 (IC95\%: 101,7 - 121,0) em 2001 para 143,1 (IC95\%: 135,0 - 151,3) em 2010. Os dois dias com menos nascimentos (Natal e Dia de Finados), apresentaram diferença estatisticamente significativa em relação aos outros dias do ano $(p<0.05)$ durante praticamente todo o período (a exceção foi o Dia de Finados em 2009). A data com o maior número de nascimentos (Dia Internacional da Mulher) apresentou diferença significativa em 2005 e 2010. O aumento no número de partos cesáreos tem tido efeito significativo na data de nascimento, o que pode trazer consequências no planejamento administrativo de instituições de saúde.

Palavras-chave Nascidos vivos, Partos cesáreos, Instituições de saúde, Data de nascimento 


\section{Introdução}

Antes apenas realizada com o objetivo de salvar a vida do feto em caso de óbito materno, cesarianas têm sido utilizadas para satisfazer um complexo grupo de necessidades e justificativas como diminuição de dor, desejo de laqueadura, preferências médicas, complicações maternas e experiências anteriores positivas em relação ao procedimento $^{1-3}$.

O Brasil é um dos países com maior proporção de partos cesáreos e esse número tem crescido rapidamente. Uma revisão realizada por $\mathrm{Pa}$ tah e Malik ${ }^{4}$ concluiu que a proporção de partos cesáreos entre os países da Organização para a Cooperação e Desenvolvimento Econômico (OCDE) é, em média, menos que a metade do verificado no Brasil, 20,8 e 46,5\%, respectivamente. Dados recentes do Departamento de Informática do SUS (DATASUS) ${ }^{5}$ mostram que a proporção de cesáreas no Brasil aumentou de 37,8\% em 2000 para 43,2\% em 2005 e 52,2\% em 2010.

Independentemente dos riscos e benefícios associados à prática ${ }^{6-9}, \mathrm{o}$ aumento do número de partos cesáreos pode afetar a demanda diária por instituições de saúde por possibilitar, em boa parte dos casos, a escolha da data do nascimento.

Em 2004, a decisão do governo australiano de fornecer uma ajuda de custo à mãe de todo nascido-vivo a partir do dia 1 de julho (informação divulgada sete semanas antes da data), fez desse dia o mais fértil dos últimos $30 \operatorname{anos}^{10}$. Um estudo de nascidos vivos na ilha de Taiwan verificou aumento de $10 \%$ no número de cesáreas em dias considerados como 'auspiciosos' segundo a astrologia chinesa tradicional ${ }^{11}$. Uma análise semelhante realizada nos EUA, encontrou aumento de $12,1 \%$ no número de cesáreas realizadas no Dia de São Valentim (14 de fevereiro) e uma diminuição de $16,9 \%$ para o Dia das Bruxas $\left(31\right.$ de outubro) ${ }^{12}$.

Estudos realizados no Brasil têm apontado para algumas consequências do aumento do número de partos cesáreos. Uma análise realizada em Minas Gerais verificou maior morbidade materna entre puérperas com parto cesariano, mas maior morbidade neonatal em recém-nascidos por via vaginal ${ }^{13}$. Um estudo de Moraes et al. ${ }^{14}$ encontrou associação positiva entre aumento no percentual de cesarianas e aumento da proporção recém-nascidos com baixo peso. Uma análise realizada em Pelotas, Rio Grande do Sul, em três períodos de tempo, verificou alta prevalência de prematuridade entre os partos cesáreos $^{15}$.
Dois estudos realizados em maternidades de Ribeirão Preto identificaram uma maior proporção de nascimentos por via cesárea durante os dias de semana (se comparados aos domingos) ${ }^{16,17}$. A diminuição do número de partos cesáreos durante os fins de semana é um resultado frequentemente verificado para a maioria dos países $^{18}$, e um indicador de algum grau de escolha da data de nascimento ${ }^{16}$.

Analisar a diferença entre as datas de nascimentos para partos vaginais e cesáreos no Brasil pode trazer não só informações sobre preferências culturais, mas também ajudar no planejamento administrativo de instituições de saúde, através da determinação do número de funcionários necessários em datas específicas ou dias da semana. A relevância para a área de saúde pública vem da possibilidade de que a preferência (ou a rejeição) por datas específicas possa contribuir para a antecipação da data de nascimento, aumentando a prevalência de prematuridade. O objetivo do presente estudo é comparar a distribuição das datas de nascimento de partos cesáreos e vaginais, utilizando dados dos últimos dez anos do Município de São Paulo.

\section{Metodologia}

Foram utilizados os dados dos 1.933 .137 nascidos vivos ocorridos no Município de São Paulo entre 2001 e 2010 (inclusive), obtidos por solicitação especial enviada ao Sistema de Informações de Nascidos Vivos (SINASC) da Prefeitura do Município de São Paulo. O período foi referente aos dez últimos anos para os quais as informações estão disponíveis. Os nascidos vivos foram informados segundo data exata de nascimento e tipo de parto (vaginal ou cesáreo), conforme presente na declaração de nascido vivo. Os 1.303 partos com tipo de procedimento 'não informado' foram excluídos da análise (1.303/ $1.933 .137=0,07 \%)$.

A análise foi dividida em duas partes: teste de diferença no número de nascimentos segundo o dia da semana (segunda-feira a domingo) e segundo a data exata do ano ( 1 janeiro a $31 \mathrm{de}$ dezembro).

O primeiro dia da semana de cada ano (no caso de 1 de janeiro de 2001, por exemplo, foi uma segunda-feira) indica o início da primeira semana deste ano. Para a análise do número médio de nascimentos segundo dia da semana todos os dias foram incluídos. No caso da comparação dos nascimentos ocorridos em domin- 
gos com os ocorridos nos outros dias da mesma semana, foram incluídas apenas as semanas completas (sete dias). Assim, os últimos dias do ano (no caso de anos bissextos, os dois últimos dias; no caso dos outros, o último dia), foram excluídos desta análise. Os testes de significância foram feitos pelo cálculo do t de Student para observações pareadas (cada domingo foi comparado com os outros dias da mesma semana). Foi adotado o nível de significância de 5\% $(\mathrm{p}<0,05)$ ao longo da pesquisa.

Para a análise da data de nascimento, foi primeiramente calculado o número médio de nascidos vivos para cada dia do ano no período de dez anos incluídos na análise. Dentro de um mesmo ano, o número médio de nascidos vivos diário foi comparado com o valor verificado para as cinco datas menos frequentes observadas no período (Natal, Dia de Finados, Véspera de Natal, Véspera de Ano Novo e Ano Novo) e para a mais frequente (Dia Internacional da Mulher), com o objetivo de testar a presença de diferença significativa, utilizando análise do desvio-padrão e intervalo de confiança de 95\%. Essa comparação foi feita separadamente para partos cesáreos, vaginais e para o total de nascimentos.

Com o objetivo de controlar pelas variações no número de nascimentos ao longo do ano (já que em São Paulo, verifica-se um aumento do número de nascimentos de janeiro a março e queda ao longo do resto do ano), as datas foram também comparadas com os nascimentos dentro de um período de duas semanas em sua vol- ta. Por exemplo, o número de nascimentos ocorridos no Natal foi comparado com os nascimentos de 18 de dezembro a 1 de janeiro. Para testar essa diferença, foi realizada uma análise de variância (ANOVA) com o objetivo de controlar pelo efeito do fim de semana na variação do número médio de óbitos. No caso do Natal: $\mathrm{Y}_{\text {Natal }}=\alpha_{0}+$ $\alpha_{1} D_{1 \text { Natal }}+\alpha_{2} D_{2}$, onde $Y_{\text {Natal }}$ é o excesso de nascimentos nesta data em relação aos outros dias, $\alpha_{0}$ é o intercepto, $\alpha_{1}$ é a diferença entre o número de óbitos esperados para o Natal e o esperado para os outros dias no intervalo de duas semanas, $\mathrm{D}_{1}$ é a variável dummy referente ao Natal, $\alpha_{2}$ é a diferença no número de nascimentos para os dias de semana (vs. fim de semana) e $\mathrm{D}_{2}$ é a variável dummy referente aos dias de semana (segunda a sexta-feira).

\section{Resultados}

Pelo Gráfico 1 é possível verificar a rápida mudança nos tipos de parto no Município de São Paulo entre 2001 e 2010. Em 2001, 51,1\% dos partos eram vaginais e 48,9\% eram cesáreos. Essa proporção se inverteu já em 2002 e, em 2010, $43,2 \%$ eram vaginais e $56,8 \%$ eram cesáreos.

O Gráfico 2 permite a análise da diferença no número médio de nascimentos segundo os dias da semana para os dois tipos de parto. Entre os partos vaginais, existe uma distribuição relativamente homogênea dos dias da semana, com uma pequena diferença em relação aos domingos (Grá-

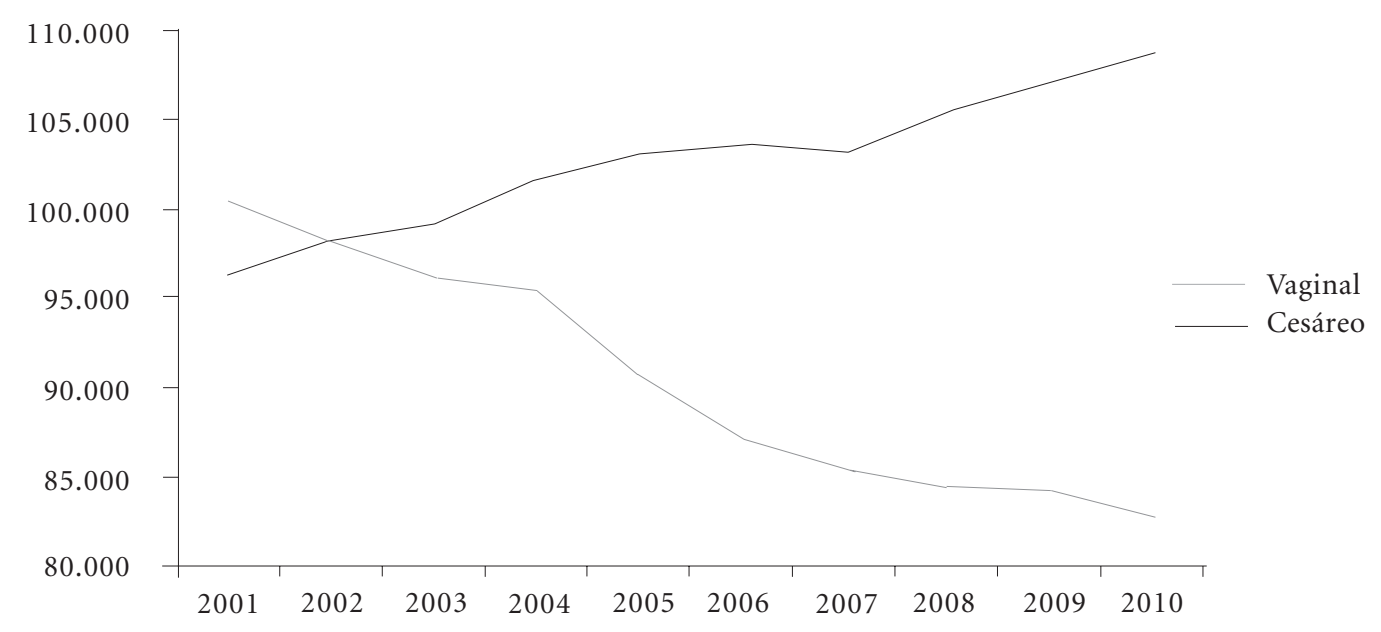

Gráfico 1. Número de nascimentos segundo tipo de parto, Município de São Paulo, Brasil, 2001 a 2010. 
fico 2a). Para os partos cesáreos verifica-se uma clara predominância de nascimentos durantes os dias de semana (segunda a sexta-feira), seguido dos sábados e dos domingos (Gráfico 2b).

$\mathrm{O}$ aumento da proporção do número de partos cesáreos em relação aos vaginais de 2001 a 2010 teve consequência direta na diminuição do número de partos ocorridos aos domingos. O Gráfico 3 mostra a diferença média entre o número de partos em domingos e os ocorridos nos outros dias da semana, com seus respectivos intervalos de confiança. Em 2001, em média, os domingos apresentavam 111,35 partos a menos que os outros dias da semana (IC95\%: 101,74 120,95); em 2010 essa diferença aumentou para 143,10 (IC95\%: 134,95 - 151,25).

A Tabela 1 analisa as cinco datas com menor número de nascimentos e a data com o maior. São elas, respectivamente: Natal ( 25 de dezembro), Dia de Finados (2 de novembro), Véspera de Ano Novo (31 de dezembro), Véspera de Natal (24 de dezembro), Ano Novo (1 de janeiro) e Dia Internacional da Mulher (8 de março). Entre as cinco datas com menor número de nascimento, quatro aumentaram a sua distância para a média diária no período de análise (sendo a Véspera de Natal a

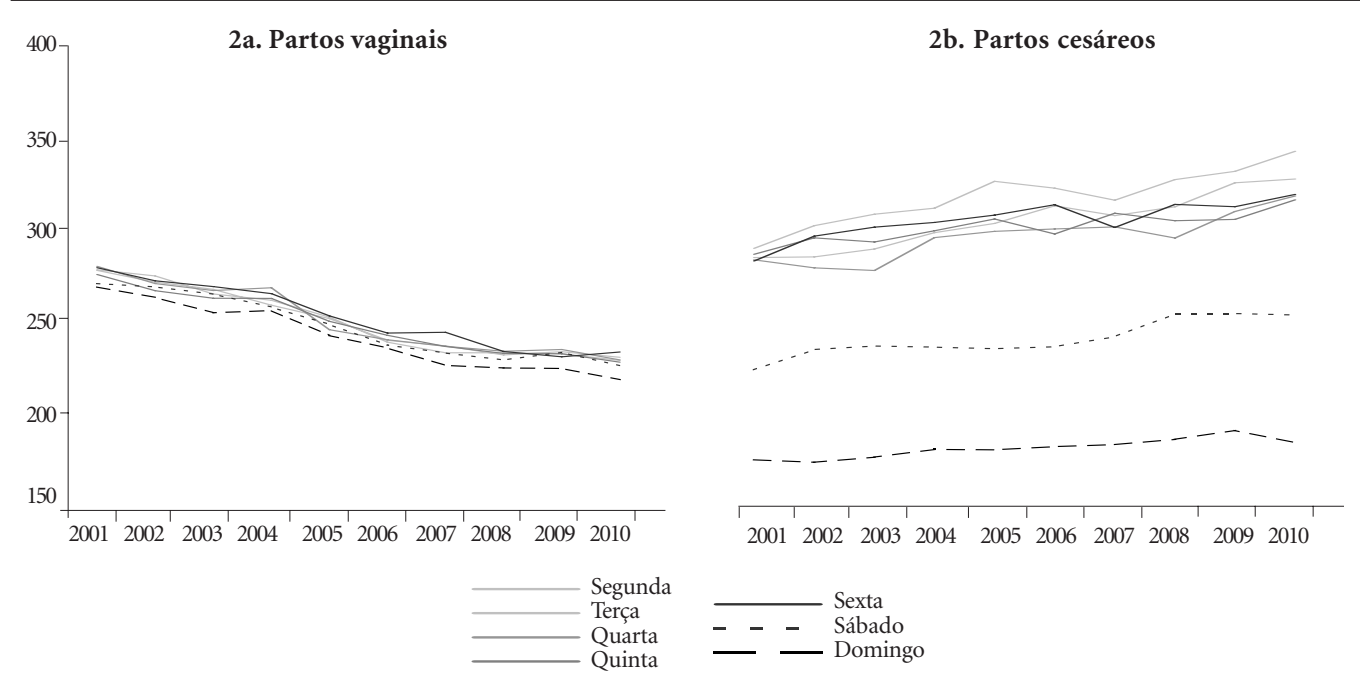

Gráfico 2. Número médio de nascimentos segundo dia da semana, Município de São Paulo, Brasil, 2001 a 2010.

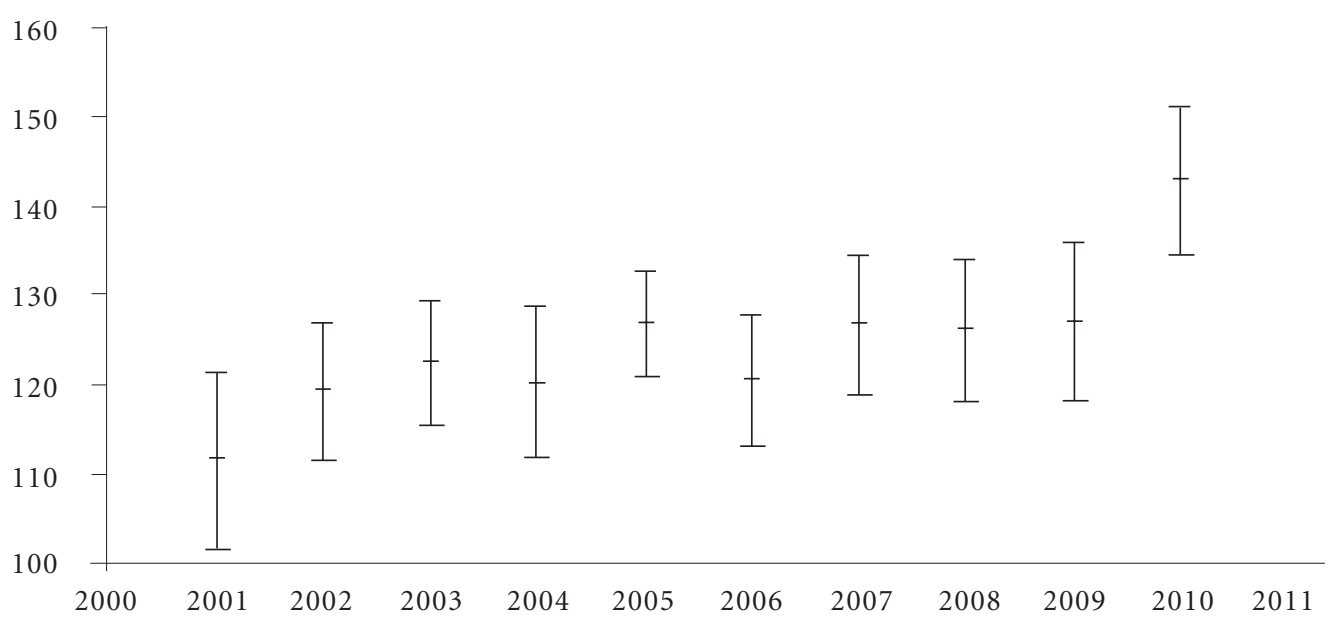

Gráfico 3. Diferença média entre o número de partos ocorridos ao domingo e os outros dias da semana (com Intervalo de Confiança de 95\%), Município de São Paulo, Brasil, 2001 a 2010 
única exceção). Em 2010, a data com menos nascimentos entre todos os tipos de parto foi o Dia de Finados (220 nascimentos a menos que a média dos outros dias). Todas as cinco datas tiveram um número de nascimento estatisticamente menor $(\mathrm{p}<0,05)$ que a média em 2010. Em relação ao Dia Internacional da Mulher, apesar de ser a data com maior número de nascimentos no período, a diferença para a média diária dos dois tipos de parto foi estatisticamente significante apenas em 2005 e 2010 (144 e 159 nascimentos a mais, respectivamente). Entre os partos vaginais, 12 das 60 datas e anos analisados apresentaram diferença estatisticamente significante, enquanto que entre partos cesáreos foram 45.

A Tabela 2 apresenta a diferença entre o número de nascimentos das datas mencionadas anteriormente e o período de duas semanas em sua volta, controlando-se pelos fins de semana. Analisando todos os partos, no dia de Natal esperam-se, em média, 141,62 nascimentos a menos que datas próximas. Apesar de ter uma diferença média esperada menor (137,97 dias), o Dia de Finados apresentou o intervalo de confiança mais estreito $(-162,39$ a -113,55), indicando menor variância (maior consistência). As cinco datas com menores nascimentos apresentaram diferença estatisticamente significante $(\mathrm{p}<0,001)$ em relação aos outros dias. No caso do Dia Internacional da Mulher, a diferença foi marginalmente significante $(\mathrm{p}=0.052)$. Entre os partos vaginais, apenas o Natal, Dia de Finados e Véspera de Natal apresentaram diferença estatisticamente significante $(\mathrm{p}<0.05)$. No caso dos partos cesáreos, a maior variabilidade diária no número de partos levou a um maior desvio padrão anual. Desse modo, uma diferença de pelo menos 120 nascimentos foi necessária para definir diferença estatisticamente significativa, enquanto que para partos vaginais foi necessária uma diferença de apenas 50 nascimentos, em média.

\section{Discussão}

O presente estudo aponta para a presença de uma rápida e consistente transição nos tipos de parto no Município de São Paulo, o que tem trazidos efeitos diretos nas datas de nascimento. $\mathrm{O}$ aumento da proporção de partos cesáreos tem levado a uma diminuição do número de partos ocorridos aos domingos e em algumas datas específicas, principalmente em relação ao Natal, Dia de Finados e Ano Novo.

De 2001 a 2010 verificou-se uma diminuição de $10,2 \%$ no número de partos ocorridos aos

Tabela 1. Diferença entre o número de nascimentos ocorridos nas cinco datas com menos nascimentos (e na data com mais) e a média diária de nascimentos, Município de São Paulo, Brasil, 2001 a 2010.

\begin{tabular}{|c|c|c|c|c|c|c|c|c|c|c|}
\hline Data & 2001 & 2002 & 2003 & 2004 & 2005 & 2006 & 2007 & 2008 & 2009 & 2010 \\
\hline \multicolumn{11}{|l|}{ Partos vaginais } \\
\hline 1 - Natal & $-60^{*}$ & -17 & $-56^{*}$ & -37 & $-70^{*}$ & 38 & -16 & -21 & -18 & 7 \\
\hline 2 - Finados & -46 & -35 & -10 & $-67^{*}$ & $-56^{*}$ & -26 & -33 & -30 & 3 & $-55^{*}$ \\
\hline 3 - Véspera de Ano Novo & -43 & -37 & -33 & $-58^{*}$ & -35 & $-52^{*}$ & -29 & 5 & -18 & -9 \\
\hline 4 - Véspera de Natal & $-61^{*}$ & -41 & -39 & -35 & -25 & -38 & -20 & -36 & -32 & -25 \\
\hline 5 - Ano Novo & -9 & -12 & -36 & -12 & -33 & -38 & -36 & -14 & -39 & -50 \\
\hline Dia Internacional da Mulher & 2 & 44 & 28 & $58^{*}$ & $96^{*}$ & 6 & 42 & 21 & 24 & 43 \\
\hline \multicolumn{11}{|l|}{ Partos cesáreos } \\
\hline 1 - Natal & $-112^{*}$ & $-143^{*}$ & $-116^{*}$ & $-137^{*}$ & $-166^{*}$ & $-144^{*}$ & $-144^{*}$ & $-151^{*}$ & $-160^{*}$ & -166 \\
\hline 2 - Finados & -97 & $-121^{*}$ & $-137^{*}$ & $-136^{*}$ & -118 & $-135^{*}$ & $-135^{*}$ & $-135^{*}$ & $-138^{*}$ & $-165^{*}$ \\
\hline 3 - Véspera de Ano Novo & $-119^{*}$ & $-124^{*}$ & $-119^{*}$ & $-136^{*}$ & $-153^{*}$ & $-155^{*}$ & $-147^{*}$ & -107 & $-122^{*}$ & $-160^{*}$ \\
\hline 4 - Véspera de & $-106^{*}$ & $-130^{*}$ & -88 & $-127^{*}$ & $-148^{*}$ & $-141^{*}$ & $-140^{*}$ & $-130^{*}$ & $-148^{*}$ & $-136^{*}$ \\
\hline 5 - Ano Novo & -82 & $-123^{*}$ & $-113^{*}$ & -105 & $-144^{*}$ & $-147^{*}$ & $-146^{*}$ & $-136^{*}$ & $-166^{*}$ & $-152^{*}$ \\
\hline Dia Internacional da Mulher & 54 & 59 & -10 & 65 & 48 & 33 & 37 & 34 & -82 & $116^{*}$ \\
\hline \multicolumn{11}{|l|}{ Total } \\
\hline 1 - Natal & $-172^{*}$ & $-161^{*}$ & $-172^{*}$ & $-174^{*}$ & $-236^{*}$ & $-172^{*}$ & $-178^{*}$ & $-182^{*}$ & $-188^{*}$ & $-209^{*}$ \\
\hline 2 - Finados & $-143^{*}$ & $-157^{*}$ & $-147^{*}$ & $-203^{\star}$ & $-174^{*}$ & $-160^{*}$ & $-168^{*}$ & $-166^{*}$ & -135 & $-220^{*}$ \\
\hline 3 - Véspera de Ano Novo & $-162^{*}$ & $-162^{*}$ & $-152^{*}$ & $-194^{*}$ & $-188^{*}$ & $-206^{*}$ & $-176^{*}$ & -103 & -140 & $-169^{*}$ \\
\hline 4 - Véspera de Natal & $-167^{*}$ & $-172^{*}$ & -127 & $-162^{\star}$ & $-173^{*}$ & $-178^{*}$ & $-160^{*}$ & $-167^{*}$ & $-180^{*}$ & $-161^{*}$ \\
\hline 5 - Ano Novo & -91 & -136 & $-149^{*}$ & -117 & $-188^{*}$ & $-184^{*}$ & $-182^{*}$ & $-151^{*}$ & $-205^{*}$ & $-202^{*}$ \\
\hline Dia Internacional da Mulher & 56 & 103 & 18 & 123 & $144^{*}$ & 40 & 79 & 55 & -58 & $159^{*}$ \\
\hline
\end{tabular}

$\mathrm{p}<0,05$ (em relação à média do respectivo ano) 


\begin{tabular}{|c|c|c|c|c|c|}
\hline \multicolumn{6}{|l|}{418} \\
\hline \multirow[t]{21}{*}{ 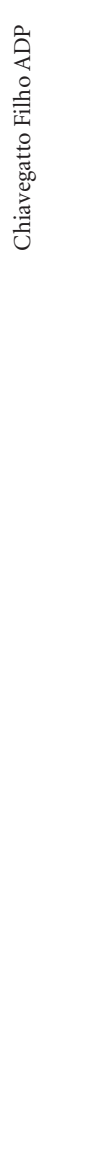 } & $\begin{array}{l}\text { Tabela 2. Diferença entre o n } \\
\text { com mais) e a média dos out } \\
\text { semana, Município de São Pa }\end{array}$ & \multicolumn{3}{|c|}{$\begin{array}{l}\text { Tabela 2. Diferença entre o número de nascimentos nas cinco datas com menos nascimentos (e na data } \\
\text { com mais) e a média dos outros dias numa janela de duas semanas, controlando-se pelo efeito dos fins de } \\
\text { semana, Município de São Paulo, Brasil }\end{array}$} & $\begin{array}{l}\text { na data } \\
\text { s fins de }\end{array}$ \\
\hline & Data & Diferença & Intervalo de cor & nfiança $95 \%$ & p-valor \\
\hline & \multicolumn{5}{|l|}{ Partos vaginais } \\
\hline & 1 - Natal & $-17,43$ & $-32,07$ & $-2,79$ & 0,02 \\
\hline & 2 - Finados & $-15,90$ & $-31,22$ & $-0,58$ & 0,04 \\
\hline & 3 - Véspera de Ano Novo & $-17,66$ & $-35,72$ & 0,39 & 0,06 \\
\hline & 4 - Véspera de Natal & $-17,52$ & $-32,23$ & $-2,81$ & 0,02 \\
\hline & 5 - Ano Novo & $-16,37$ & $-34,43$ & 1,69 & 0,08 \\
\hline & $\begin{array}{l}\text { Dia Internacional da Mulher } \\
\text { Partos cesáreos }\end{array}$ & 10,98 & $-6,29$ & 28,26 & 0,21 \\
\hline & 1 - Natal & $-120,67$ & $-177,43$ & $-63,91$ & $<0.001$ \\
\hline & 2 - Finados & $-122,07$ & $-143,09$ & $-101,04$ & $<0.001$ \\
\hline & 3 - Véspera de Ano Novo & $-104,14$ & $-157,06$ & $-51,22$ & $<0.001$ \\
\hline & 4 - Véspera de Natal & $-125,39$ & $-175,71$ & $-75,06$ & $<0.001$ \\
\hline & 5 - Ano Novo & $-105,79$ & $-157,20$ & $-54,39$ & $<0.001$ \\
\hline & $\begin{array}{l}\text { Dia Internacional da Mulher } \\
\text { Total }\end{array}$ & 12,05 & $-9,91$ & 34,03 & 0,28 \\
\hline & 1 - Natal & $-141,62$ & $-203,25$ & $-80,00$ & $<0.001$ \\
\hline & 2 - Finados & $-137,97$ & $-162,39$ & $-113,55$ & $<0.001$ \\
\hline & 3 - Véspera de Ano Novo & $-121,80$ & $-181,79$ & $-61,80$ & $<0.001$ \\
\hline & 4 - Véspera de Natal & $-142,90$ & $-197,88$ & $-87,93$ & $<0.001$ \\
\hline & 5 - Ano Novo & $-122,16$ & $-180,03$ & $-64,30$ & $<0.001$ \\
\hline & Dia Internacional da Mulher & 23,04 & $-0,21$ & 46,30 & 0,052 \\
\hline
\end{tabular}

domingos, enquanto para os outros dias da semana a diminuição foi de apenas 1,7\%. Com isso, a diferença média entre o número de nascidos no domingo em relação aos outros dias da mesma semana foi de 143 em 2010.

O presente estudo permitiu identificar algumas datas que podem ser consideradas simbólicas para os paulistanos. Verificou-se uma alta rejeição para o Natal e Dia de Finados, sendo esses os dois dias do ano com o menor número de nascimentos durante o período analisado. Com o aumento do número de partos cesáreos, essa diferença em relação aos outros dias tem aumentado. Durante todo o período analisado, a diferença entre essas datas e os outros dias do ano foi sempre estatisticamente significante para o total de nascimentos $(\mathrm{p}<0.05)$.

Apesar da sazonalidade no número de nascimentos ao longo do ano, isso não explicou o baixo número de nascimentos no Natal e Dia de Finados. Quando comparados aos dias próximos (dentro de uma janela de duas semanas), o número de nascimentos nessas datas foi estatisticamente menor aos outros, mesmo controlando-se pelo efeito do sábado e domingo $(\mathrm{p}<0.01)$.

Por outro lado, não se verificou uma preferência clara por nenhuma data do ano. O dia com o maior número de nascimentos no perío- do (Dia Internacional da Mulher, 8 de março) apresentou diferença estatisticamente significativa em relação aos outros dias para apenas 2 dos 10 anos analisados pela pesquisa. Pode-se concluir, portanto, que o efeito dos partos cesáreos na escolha da data de nascimento é mais importante para a rejeição de datas específicas do que para preferência a outras.

A diferença estatisticamente significante encontrada para algumas datas em partos vaginais, apesar de menor que as encontradas para partos cesáreos, pode ser explicada por dois motivos. Em primeiro lugar, partos vaginais apresentaram menor variabilidade diária no número de partos, levando a um menor desvio padrão anual. Assim, uma diferença diária de apenas 50 nascimentos foi considerada estatisticamente significante para partos vaginais, enquanto que para partos cesáreos foi necessária uma diferença de pelo menos 120 nascimentos, em média.

Em segundo lugar, a atual indicação de "partos vaginais" presente na declaração de nascidos vivos utilizada no Brasil pode ser um fator limitante na interpretação dos resultados do estudo. Ao não diferenciar parto natural de parto induzido, os dados oficiais podem dificultar a análise da presença de escolha da data de nascimentos ${ }^{19}$. De modo semelhante, cesáreas nem sempre são 
realizadas com planejamento prévio, principalmente no caso de urgência devido a risco fetal ou materno ${ }^{20}$. Assim, uma comparação inadequada poderia ser feita entre partos vaginais e partos cesáreos, definindo os primeiros como ocorrendo sempre de forma natural (onde a data de nascimento se encontra fora do controle materno e médico) e os outros como sendo sempre planejados, o que não é necessariamente o caso.

Outra possível limitação do estudo é o uso de dados secundários, obtidos pelo SINASC. Apesar da melhoria da qualidade de informação nos últimos anos, a declaração de nascido vivo ainda não é sempre preenchida corretamente ${ }^{21-23}$. Entretanto, estudos mostram que dados sobre o tipo de parto (usados pelo presente estudo) apresentam confiabilidade quase perfeita ${ }^{24}$.

O presente estudo aponta para uma necessidade de mudança no planejamento das maternidades, diminuindo o número de funcionários aos domingos e em determinados dias do ano. Essa mudança, entretanto, deverá ser mais importante para o caso de hospitais privados ${ }^{25,26}$. Um estudo realizado no Rio de Janeiro verificou que em hospitais públicos a taxa de cesárea foi de 33,5\%, enquanto que em privados foi de $87,4 \%{ }^{27}$.

Outro problema que pode decorrer da existência de uma forte rejeição por datas específicas é o aumento do número de nascimentos prematuros. Neste caso, os pais poderão decidir anteci-

\section{Agradecimentos}

À Eliana de Aquino Bonilha, da Gerência do SINASC-SP, por ter fornecido os dados utilizados por este artigo. À FAPESP pelo financiamento. par a data de nascimento para não arriscar que ocorra em uma data com alta rejeição. Apesar de não testada diretamente pelo estudo, essa possibilidade não pode ser descartada, principalmente levando em consideração a associação entre partos cesáreos e maior número de nascidos vivos prematuros e com baixo peso ${ }^{14,15}$.

O aumento no número de partos cesáreos nas últimas décadas não é uma característica apenas do Brasil, mas a sua a proporção encontra-se atualmente entre as maiores do mundo ${ }^{28,29}$. Esse crescimento tem sido constante para a maioria dos países, e não existe uma expectativa de reversão dessa tendência ${ }^{30}$. De fato, algumas das causas frequentemente associadas com esse aumento (possibilidade de diminuição das dores de parto e maior controle médico do procedimento $)^{1-3}$, tendem a se tornarem mais importantes com a ampliação dos sistemas de saúde, principalmente em países em desenvolvimento ${ }^{31}$.

A análise do Município de São Paulo é importante por ser atualmente o município brasileiro com o maior número de partos cesáreos. Apesar de algumas estratégias bem-sucedidas, como a implantação de Centros de Partos Normais $(\mathrm{CPN})^{32,33}$, a proporção de partos cesáreos tem aumentado rapidamente. Os resultados encontrados podem servir como exemplo do que se pode esperar para o resto do país, e mesmo para outros países, em relação aos efeitos futuros dessa mudança.

\section{Referências}

1. Dias MAB, Domingues R, Pereira APE, Fonseca SC, Gama SGN, Theme F. Trajetória das mulheres na definição pelo parto cesáreo: estudo de caso em duas unidades do sistema de saúde suplementar do estado do Rio de Janeiro. Cien Saude Colet 2008; 13(5):1521-1534.

2. Barbosa GP, Giffin K, Angulo-Tuesta A, de Souza Gama A, Chor D, D’Orsi E, dos Reis ACGV. Parto cesáreo: quem o deseja? Em quais circunstâncias? Cad Saude Publica 2003; 19(6):1611-1620.

3. Sakae TM, Freitas PF, d'Orsi E. Fatores associados a taxas de cesárea em hospital universitário. Rev Saude Publica 2009; 43(3):472-480.

4. Patah LEM, Malik AM. Modelos de assistência ao parto e taxa de cesárea em diferentes países. Rev Saude Publica 2011; 45(1):185-194.

5. Departamento de Informática do SUS (DATASUS). Informações de Saúde. [acessado 2012 out 9]. Disponível em: http://www2.datasus.gov.br/DATASUS/ index.php 
6. Hansen AK, Wisborg K, Uldbjerg N, Henriksen TB. Risk of respiratory morbidity in term infants delivered by elective caesarean section: cohort study. BMJ 2008; 336(7635):85-87.

7. Cardwell C, Stene L, Joner G, Cinek O, Svensson J, Goldacre M, Parslow R, Pozzilli P, Brigis G, Stoyanov D. Caesarean section is associated with an increased risk of childhood-onset type 1 diabetes mellitus: a meta-analysis of observational studies. Diabetologia 2008; 51(5):726-735

8. Bager P, Wohlfahrt J, Westergaard T. Caesarean delivery and risk of atopy and allergic disesase: meta analyses. Clinical \& Experimental Allergy 2008; 38(4):634-642.

9. Goldani HAS, Bettiol H, Barbieri MA, Silva AAM, Agranonik M, Morais MB, Goldani MZ. Cesarean delivery is associated with an increased risk of obesity in adulthood in a Brazilian birth cohort study. Am J Clin Nutr 2011; 93(6):1344-1347.

10. Gans JS, Leigh A. Born on the first of July: An (un) natural experiment in birth timing. J Public Econ 2009; 93:246-263.

11. Lo JC. Patients' attitudes vs. physicians' determination: implications for cesarean sections. Soc Sci Med 2003; 57(1):91-96

12. Levy BR, Chung PH, Slade MD. Influence of Valentine's Day and Halloween on Birth Timing. Soc Sci Med 2011; 73(8):1246-1248.

13. Cardoso PO, Alberti LR, Petroianu A. Morbidade neonatal e maternas relacionada ao tipo de parto. Cien Saude Colet 2010; 15(2):427-435.

14. Moraes AB, Zanini RR, Giugliani ERJ, Riboldi J. Tendência da proporção de baixo peso ao nascer, no período de 1994-2004, por microrregião do Rio Grande do Sul, Brasil: uma análise multinível. Cad Saude Publica 2011; 27(2):229-240.

15. Barros FC, Victora CG, Matijasevich A, Santos IS Horta BL, Silveira MF, Barros AJD. Preterm births, low birth weight, and intrauterine growth restriction in three birth cohorts in Southern Brazil: 1982, 1993 and 2004. Cad Saude Publica 2008; 24(Supl. 3):s390-s398.

16. Almeida S, Bettiol H, Barbieri MA, Silva AAM, Ribeiro VS. Significant differences in cesarean section rates between a private and a public hospital in Brazil. Cad Saude Publica 2008; 24(12):2909-2918.

17. Gomes UA, Silva AA, Bettiol H, Barbieri MA. Risk factors for the increasing caesarean section rate in Southeast Brazil: a comparison of two birth cohorts, 1978-1979 and 1994. Int J Epidemiol 1999; 28(4):687-694.

18. Lerchl A. Where are the Sunday babies? III. Caesarean sections, decreased weekend births, and midwife involvement in Germany. Naturwissenschaften 2008; 95(2):165-170.

19. Goldenberg RL, Culhane JF, Iams JD, Romero R. Epidemiology and causes of preterm birth. Lancet 2008; 371(9606):75-84.

20. Giglio MRP, França E, Lamounier JA. Evaluation of the quality of care for normal delivery. Rev Bras Ginecol Obstet 2011; 33(10):297-304.

21. Romero DE, Cunha CBD. Avaliação da qualidade das variáveis epidemiológicas e demográficas do Sistema de Informações sobre Nascidos Vivos, 2002. Cad Saude Publica 2007; 23(3):701-714
22. Mello Jorge M, Laurenti R, Gotlieb SLD. Análise da qualidade das estatísticas vitais brasileiras: a experiência de implantação do SIM e do SINASC. Cien Saude Colet 2007; 12(3):643-654.

23. Luquetti DV, Koifman RJ. Qualidade da notificação de anomalias congênitas pelo Sistema de Informações sobre Nascidos Vivos (SINASC): estudo comparativo nos anos 2004 e 2007. Cad Saude Publica 2010; 26(9):1756-65.

24. Theme Filha MM, Gama SGN, Cunha CB, Leal MC. Confiabilidade do Sistema de Informações sobre Nascidos Vivos Hospitalares no Município do Rio de Janeiro, 1999-2001. Cad Saude Publica 2004; 20(Supl.):S83-S91.

25. Mandarino NR, Chein MBC, Monteiro Júnior FC, Brito LMO, Lamy ZC, Nina VIS, Mochel EG, Figueiredo Neto JA. Aspectos relacionados à escolha do tipo de parto: um estudo comparativo entre uma maternidade pública e outra privada, em São Luís, Maranhão, Brasil. Cad Saude Publica 2009; 25(7):1587-1596.

26. Meller FDO, Schäfer AA. Fatores associados ao tipo de parto em mulheres brasileiras: PNDS 2006. Cien Saude Colet 2011; 16(9):3829-3835.

27. Leal MDC. Cesarianas desnecessárias: causas, conseqüências e estratégias para sua redução. Rio de Janeiro: Fundação Oswaldo Cruz; 2007.

28. Rebelo F, Rocha CM, Cortes TR, Dutra CL, Kac G. High cesarean prevalence in a national population based study in Brazil: the role of private practice. Acta Obstet Gynecol Scand 2010; 89(7):903-908.

29. Potter JE, Hopkins K, Faúndes A, Perpétuo I. Women's autonomy and scheduled cesarean sections in Brazil: a cautionary tale. Birth 2008; 35(1):33-40.

30. Stanton CK, Holtz SA. Levels and trends in cesarean birth in the developing world. Stud Fam Plann 2006; 37(1):41-48.

31. Ronsmans C, Holtz S, Stanton C. Socioeconomic differentials in caesarean rates in developing countries: a retrospective analysis. Lancet 2006; 368(9546): 1516-1523.

32. Osava RH, Silva FMB, Tuesta EF, Oliveira SMJV, Amaral MCE. Caracterização das cesarianas em centro de parto normal. Rev Saude Publica 2011; 45(6):1036-1043.

33. Lobo SF, Oliveira SMJV, Schneck CA, Silva FMB, Bonadio IC, Riesco MLG. Resultados maternos e neonatais em centro de parto normal peri-hospitalar e hospital. Rev Saude Publica 2012; 46(1): 77-86.

Artigo apresentado em 06/10/2012

Aprovado em 21/10/2012

Versão final apresentada em 26/10/2012 